\title{
Assessing Performance of Aerobic Routines using Background Subtraction and Intersected Image Region
}

\author{
John F. \\ Faculty of Computer Science \& \\ Information Technology, \\ Universiti Malaysia Sarawak Kota \\ Samarahan, Sarawak, Malaysia. \\ 46826@ siswa.unimas.my
}

\author{
Hipiny I. \\ Faculty of Computer Science \& \\ Information Technology, \\ Universiti Malaysia Sarawak \\ Kota Samarahan, Sarawak, Malaysia. \\ mhihipni@unimas.my
}

\author{
Ujir H. \\ Faculty of Computer Science \& \\ Information Technology, \\ Universiti Malaysia Sarawak \\ Kota Samarahan, Sarawak, Malaysia. \\ uhamimah@unimas.my
}

\begin{abstract}
It is recommended for a novice person to engage trained personnel before starting an unfamiliar aerobic or weight routine to gain real-time expert feedbacks. This greatly reduces the risk of injury and maximise physical gains. We present a simple image similarity measure based on intersected image region to assess a subject's performance of an aerobic routine. The method is implemented inside an Augmented Reality (AR) desktop app that employs a single RGB camera to capture still images of the subject as he or she progresses through the routine. The backgroundsubtracted body pose image is compared against the exemplar image (i.e., AR template) at specific intervals. Based on a limited dataset, our pose matching function managed an accuracy of $93.67 \%$.
\end{abstract}

Keywords-Augmented Reality (AR), Background subtraction, Image similarity measures

\section{INTRODUCTION}

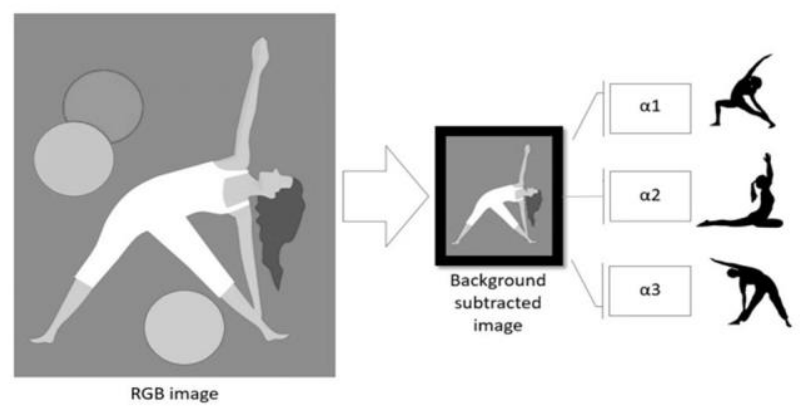

Fig. 1. Our proposed framework.

According to [1], the worldwide revenue in fitness segment encompassing two product categories: portable, connected fitness devices (i.e., wearables) and digital fitness and nutrition apps is amounting to US\$16,857 millions in 2019. With the growing interest in personal fitness and the budding trend of exercising anytime and anywhere, there is a need for a software-based solution to measure user's performance. Ideally, the measurement has to be marker-less since the use of multiple body-worn sensors is cumbersome and costly. This solution replaces the role of a human coach in both assessing and guiding the subject as he or she performs an aerobic or weight routine.
It is vital for the routine to be performed correctly to prevent and reduce the risk of injury.

978-1-7281-6593-6/19/\$31.00 @2019 IEEE

Moreover, by maintaining a correct posture throughout the routine greatly increases its effectiveness.

We use a simple image similarity measure, implemented inside a desktop AR app, to measure a subject's performance of an aerobic routine. The desktop app employs a single RGB camera as the solitary input to capture the subject's body pose at specific intervals. The exemplar (body pose) image, i.e., the AR template, is then superimposed over the subject's background-subtracted body pose image. The ratio of the intersected region to the union region determines the similarity score. Thus, a higher similarity score correlates to a better performance of the routine. See Figure 1 for a

visualisation of our proposed framework.

Computer vision approaches have been used to recognise human activities [2, 3, 4 and 5], and measuring task performance, e.g., [6] and [7]. Models are either learned from image or motion-based features. We adopt a similar approach in matching the subject's body pose to known exemplars by measuring the image similarity. The similarity score is then used to determine how well the subject had matched the required body posture at different stages of the aerobic routine.

\section{RELATED WORK}

\section{A. Fitness mobile apps}

The following mobile apps use static videos/animated gifs to demonstrate an aerobic or weight routine to the user. No measurement, except for time-to-completion, is made since these apps are not paired with any sensors.

Runtastic Result [8] is an app available on both Android and iOS platform. The app provides a series of workouts for the user aiming to either lose weight, build muscle mass or improve stamina. The app provides purchasable weekly training plans. The workouts are in the form of downloadable videos. Each video contains a countdown timer and a voice 